\title{
Two Nucleoporin98 homologous genes jointly participate in the regulation of starch degradation to repress senescence in Arabidopsis
}

Long Xiao ${ }^{1 \dagger}$, Shanshan Jiang ${ }^{2 \dagger}$, Penghui Huang ${ }^{2 \dagger}$, Fulu Chen ${ }^{2}, X_{\text {u Wang }}^{2}$, Zhiyuan Cheng ${ }^{2}$, Yuchen Miao ${ }^{3}$, Liangyu Liu ${ }^{4}$, Iain Searle ${ }^{5}$, Chunyan Liu ${ }^{1}$, Xiao-Xia Wu ${ }^{1}$, Yong-Fu Fu ${ }^{2 *+} \mathbb{D}$, Qingshan Chen ${ }^{1+}$ and Xiao-Mei Zhang ${ }^{2+}$

\begin{abstract}
Background: Starch is synthesized during daylight for temporary storage in leaves and then degraded during the subsequent night to support plant growth and development. Impairment of starch degradation leads to stunted growth, even senescence and death. The nuclear pore complex is involved in many cellular processes, but its relationship with starch degradation has been unclear until now. We previously identified that two Nucleoporin98 genes (Nup98a and Nup986) redundantly regulate flowering via the CONSTANS (CO)-independent pathway in Arabidopsis thaliana. The double mutant also shows severe senescence phenotypes.

Results: We find that Nucleoporin 98 participates in the regulation of sugar metabolism in leaves and is also involved in senescence regulation in Arabidopsis. We show that Nup98a and Nup98b function redundantly at different stages of starch degradation. The nup98a-1 nup98b-1 double mutant accumulates more starch, showing a severe early senescence phenotype compared to wild type plants. The expression of marker genes related to starch degradation is impaired in the nup98a-1 nup986-1 double mutant, and marker genes of carbon starvation and senescence express their products earlier and in higher abundance than in wild type plants, suggesting that abnormalities in energy metabolism are the main cause of senescence in the double mutant. Addition of sucrose to the growth medium rescues early senescence phenotypes of the nup98a-1 nup98b-1 mutant.

Conclusions: Our results provide evidence for a novel role of the nuclear pore complex in energy metabolism related to growth and development, in which Nup98 functions in starch degradation to control growth regulation in Arabidopsis.
\end{abstract}

Keywords: Nup98, Starch, Sugar, Senescence, Nuclear pore complex, Nucleoporin

\footnotetext{
* Correspondence: fufu19cn@163.com

†Long Xiao, Shanshan Jiang, and Penghui Huang contributed equally to this work.

${ }^{\dagger}$ Yong-Fu Fu, Qingshan Chen, and Xiao-Mei Zhang shared correspondence equally.

${ }^{2}$ MOA Key Lab of Soybean Biology (Beijing), National Key Facility of Crop Gene Resource and Genetic Improvement, Institute of Crop Sciences,

Chinese Academy of Agricultural Sciences, Nandajie 12, Zhongguancun, Haidian District, Beijing 100081, China

Full list of author information is available at the end of the article
} 


\section{Background}

The nuclear pore complex (NPC) is the key bridge for communication of macromolecules between the nucleus and cytoplasm and it regulates gene expression [1]. NPC is built by at least 30 unique nucleoporins (Nups), which are highly conserved in eukaryotic cells $[2,3]$. Nup98 is a mobile and peripheral Phe-Gly domain (FG)-containing nucleoporin which spans from the nucleus and cytoplasmic parts of the central channel of the NPC $[4,5]$. Arabidopsis thaliana Nup98a (also known as DRACULA2, DRA2) is also found in different subcellular locations [6]. Nup98 is involved in the regulation of cargo export and import, gene expression, transcriptional memory, and multiple developmental processes in animals and yeast [4-9]. In Arabidopsis, DRA2 regulates the shade avoidance syndrome [6]. In rice, the Nup98 homolog APIP12 (AVRPIZ-T INTERACTING PROTEIN12) is involved in basal resistance against the pathogen Magnaporthe oryzae and targeted by the Magnaporthe effector AvrPiz-t [10]. In a recent report in Arabidopsis, the interaction between the proteins Nup98 and Nup88/MOS7 (MODIFIER OF SNC1,7) is required for plant immunity against the necrotrophic fungal pathogen Botrytis cinerea and mitogen-activated protein kinase signalling [11]. We found that Nup98 contributes to flowering regulation in Arabidopsis [12].

Senescence is an important cellular process associated with various developmental and environmental cues [13]. Before death, plants remobilize resources in senescing organs and translocate them to sink organs to support their growth and development [14-16]. To date, at least 200 genes have been identified that regulate, or participate in, senescence processes in plants. Starch, a main photosynthetic product synthesized in leaves, is degraded at night to support plant development. Impairment of gene function during different steps in starch degradation hinders plant growth to different extents $[17,18]$. Sugar directly or indirectly regulates senescence: sugar accumulation not only triggers and accelerates, but may also delay senescence [19-21]. In fact, in response to sugar, senescence process in plants is ageor condition-dependent [20,22, 23]. Within the sugar pathway, three key genes have been identified: glucose sensor HEXOKINASE 1 (HXK1), energy sensor PROTEIN KINASE10 (KIN10) and KIN11, and TOR (THE TARGET OF RAPAMYCIN) [21, 24-27].

Currently, the mechanism of NPC in regulation of starch degradation and senescence in plants is still unknown. However, in animals there are several studies reporting that NPC controls cell senescence through modifying chromosome structure, DNA repair and replication or cell division [28-32]. Impairment of NPCs results in dysfunction of nucleo-cytoplasm transportation [30]. Both Nup107 [33] and Tpr (TRANSLOCATED
PROMOTER REGION) [34, 35] have been linked to cancer cell proliferation and cellular senescence in aging cells.

In this study, we focused on two Nup98 homologous genes, Nup98a and Nup98b, in Arabidopsis thaliana. Single mutants of either of these genes in Arabidopsis have no obvious flowering and senescence phenotypes. However, the double mutant, nup $98 a-1$ and nup $98 b-1$, show significant early-senescence phenotypes. Gene expression analysis demonstrates that Nup $98 a$ and Nup $98 b$ participate in starch degradation conferring growth regulation. Further analysis suggests that early senescence in the double mutant may result from a defect in the initial steps of starch degradation and result in a dysfunction in energy supply. Interestingly, the early senescence phenotype in this double mutant can be rescued by the addition of sugar to the growth medium. Our data suggest that Nup98a and Nup98b might function redundantly in regulation of starch degradation and contribute to normal growth and development in Arabidopsis.

\section{Results}

Nup98 mutation results in early senescence in Arabidopsis thaliana

Nup98 is a highly conserved nuclear pore protein in eukaryotes. In Arabidopsis thaliana, there are two homologs of mammalian Nucleoporin98 - Nup98a (At1g10390) and Nup98b (At1g59660), which share highly conserved amino acid sequences in the Phe-Gly (FG)-repeats and autoproteolytic domain (APD, Supplementary Figure 1) $[3,6]$. Nup 98 a was previously reported as DRACULA2 (DRA2), a regulator of the shade avoidance syndrome (SAS) in Arabidopsis [6] and immune responses to a rice fungal pathogen [11]. To investigate Nup98 functions in plant development, we screened mutants of nup98a (SALK_080083, SALK_ 090744, SALK_023493, SALK_103803, SALK_015016 ordered from ABRC) and nup $98 b$ (CS803848 and GABI 288A08 ordered from GABI T-DNA mutant center). Homozygous lines were isolated for the following insertional mutants of SALK_103803, SALK_015016, GABI_288A08, and among them SALK_103803 and GABI_288A08 were the mutants reported by Parry [9]. The T-DNAs in these homozygous mutants are inserted in protein-encoding regions (Fig. 1a) and RT-PCR demonstrated that these mutants were null alleles (Fig. 1b, Supplementary Figure 2), consistent with Parry's results [9]. As in previous studies [9], we did not observe any obvious phenotypes in flowering and senescence in either the nup $98 a$ or nup $98 b$ single mutants compared to wild type plants under long-day photoperiod conditions (Fig. 1c). As Nup98a and Nup98b share highly conserved amino acid sequences (Supplementary Figure 1), we 


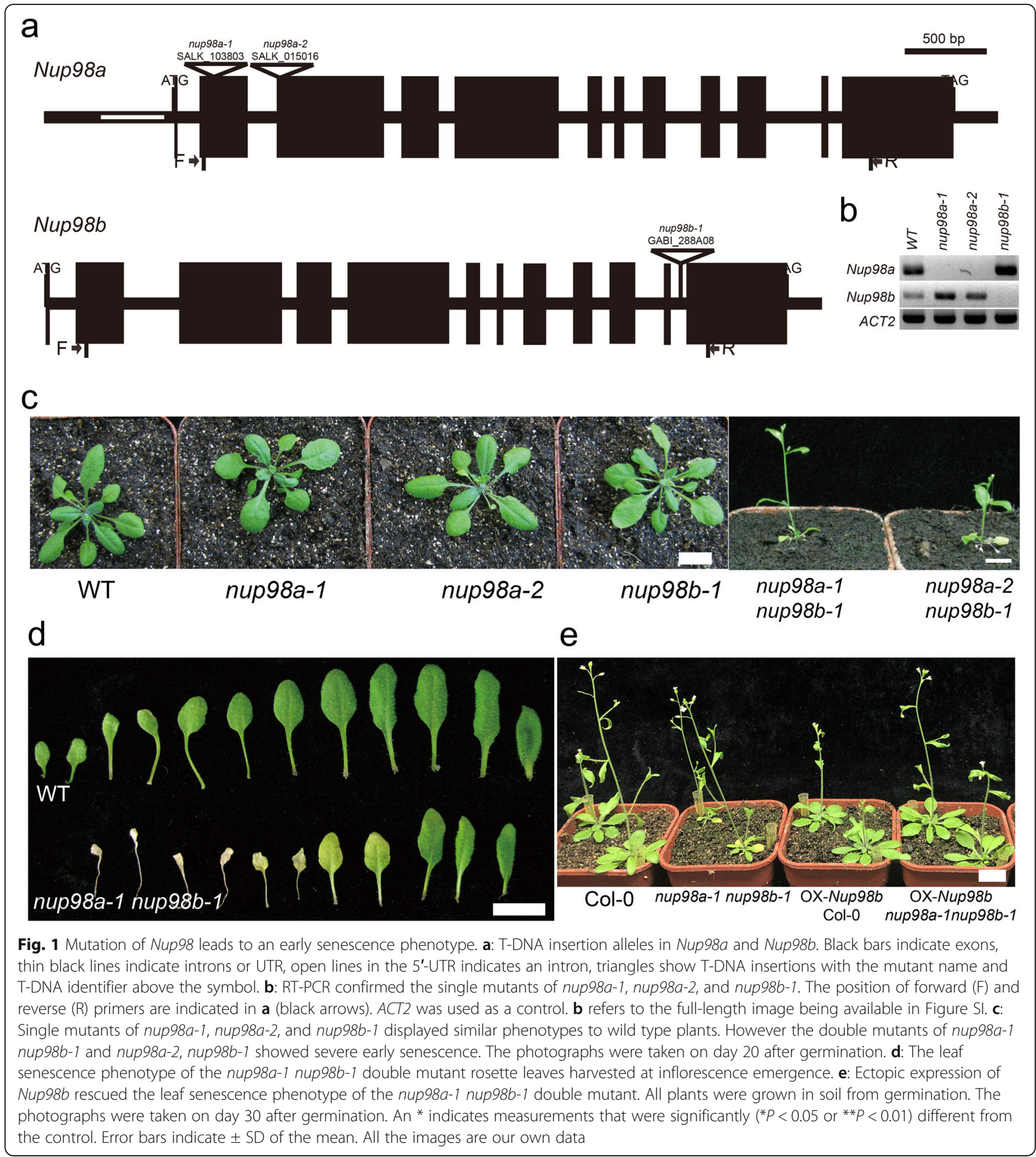

tested the hypothesis that Nup98a and Nup98b function redundantly. Strikingly, two double mutants, nup $98 a-1$ nup $98 b-1$ and nup98a-2, nup 98b-1, displayed similar early senescence phenotypes when compared with wild type plants (Fig. 1c and d). The senescence phenotype appeared not only in leaves, but also in the whole plant. Also, the double mutant plants had additional phenotypes, including smaller inflorescences, flowers and siliques, short stature, and severe sterility when compared with wild type plants (Supplementary Figures 3 and 4). We recently reported that the nup $98 a-1$ nup $98 b-1$ double mutants have an early flowering phenotype [12]. Expressing the Nup98b gene in the double mutant, rescued the senescence phenotypes (Fig. 
1e). These results confirm that Nup98a and Nup98b act redundantly.

To investigate whether the senescence phenotype is specific to the nup $98 a-1$ nup $98 b-1$ mutant, we selected another three nucleoporin mutants, nup 96-1, nup160-1, and nup107-1, which showed flowering phenotypes in our previous report [36], to analyze the effect of other nuclear pore components on senescence. To our surprise, no early senescence phenotypes were observed in these mutants (Supplementary Figure 5), suggesting that some of nucleoporins may not be involved in the regulation of senescence and that Nup98 may have specific functions in senescence regulation.

\section{The Nup98 gene is involved in multiple pathways of senescence initiation}

Because the early senescence observed in the nup $98 a-1$ nup 98b-1 double mutant could be a secondary effect of altered development, we further explored the role of Nup $98 a$ and Nup $98 b$ in senescence regulation. To date, at least 200 genes have been identified to participate in senescence regulation in plants [37]. We summarized main literatures as shown in Supplementary Figure 6, which shows that various endogenous and environmental cues, such as hormones, sugar, light and photoperiod, and stresses, trigger plant senescence in multiple crosstalking patterns. To identify the potential link to early senescence, we measured mRNA abundance of important senescence-associated genes in the nup $98 a-1$ nup $98 b$-1 double mutant plants (Supplementary Figure 6) at ZT0 (Zeitgeber 0, the time when light turned on) and ZT16 (the time when light turned off) in plants grown under long day conditions by real time quantitative PCR (RT-qPCR). As shown in Fig. 2, gene expressions in the nup98a-1 nup $98 b-1$ double mutant were significantly different from that in wild type plants. In the first category, WRKY53 (encoding WRKY DNABINDING PROTEIN53), SAG13 (encoding SENES CENCE-ASSOCIATED GENE13), WRKY6, NAC1 (encoding NAM, ATAF, and CUC) and NAC2 displayed higher transcript abundances at both ZT0 and ZT16 (Fig. 2a), suggesting that genes in the stress pathway (WRKY53, WRKY6, and NAC1) and SA pathway (WRKY53, WRKY6, and NAC2) were related to the senescence phenotypes of the nup $98 a-1$ nup $98 b-1$ double mutant. In the second category, SAG12, NAP (encoding NAC DOMAIN CONTAINING PROTEIN), SAG2 and CAT1 (encoding CATALASE1), also genes in stress and SA pathways, were only increased in abundance at either ZT0 or ZT16 (Fig. 2b). In contrast, in the third category, SAUR36 (encoding SMALL AUXIN UPREGULATED36), WRKY70, ARP4 (encoding ACTIN-RELATED PROTEIN4), SEN1 (encoding SPLICING ENDONUCLEASE 1), and COI1 (encoding CORONATINE INSENSITIVE1) were decreased in abundance at either ZT0 and/or ZT16 (Fig. 2c), indicating that auxin (SAUR36) and jasmonate (COI1) signalling may be negatively related to the nup 98 senescence phenotypes. The abundance of AGL15 (encoding AGAMOUS-LIKE15), EBP1 (encoding ERBB-3 BINDING PROTEIN1), RPS6a (encoding RIBOSOMAL PROTEIN6a), and NPR1 (encoding NONEXPRESSOR OF PR-GENES1) had the opposite changes at ZT0 and ZT16 (Fig. 2d), suggesting that the function of these genes on Nup98 senescence regulation may be dependent on the circadian clock.

This expression profile suggests that there are at least three senescence processes affected in the nup 98 double mutant. Firstly, several pathways, mainly the stress and SA pathways, were involved in regulation of senescence in the nup 98a-1 nup 98b-1 double mutant. Secondly, different genes function in their own special modes by being positively or negatively regulated at different phases (morning or afternoon phases). Thirdly, some genes, such as WRKY53, WRKY6, NAP1, and SAG2, may play roles in multiple pathways. The results indicate that the senescence phenotypes of the nup 98 double mutant involve multiple pathways. Many genes showed circadian expression patterns, consistent with our previous report that Nup 98 genes are regulated by processes involving the circadian clock [12].

\section{Starch metabolism is impaired in the nup98a-1 nup98b-1 double mutant}

During photosynthesis, starch is synthesized and stored in chloroplasts and then degraded at night. A number of genes are involved in starch degradation in plants (Supplementary Figure 7) $[17,18]$. Firstly, we checked starch homeostasis in leaves of the nup $98 a-1$ nup $98 b-1$ double mutant plants grown under $12 \mathrm{~h}$ light $/ 12 \mathrm{~h}$ dark conditions (at ZT0, dawn, and ZT12, dusk) (Fig. 3). Leaves of all plants accumulated starch at dusk, but double mutant plants had much more starch than wild type plants as determined by both iodine staining (Fig. 3a) and starch quantitative assays (Fig. 3b). The more intense signals in older (21 and 28-day) leaves in the double mutant may be a consequence of accumulating starch over time. Therefore, the double mutant accumulated much more starch, not only at dawn but also at dusk, than wild type plants, and this phenotype was much clearer in 14- and 21-day-olds seedlings (Fig. 3a and b). Together, these results suggest that starch metabolism is impaired in the nup 98a-1 nup 98b-1 double mutant.

We measured the transcript abundance of genes involved in starch metabolism [17] by RT-qPCR (Fig. 4) and found that many genes had significantly lower RNA accumulation in the double mutant at least at one time point when compared with wild type plants. These genes encode enzymes for the degrading process of starch, not 


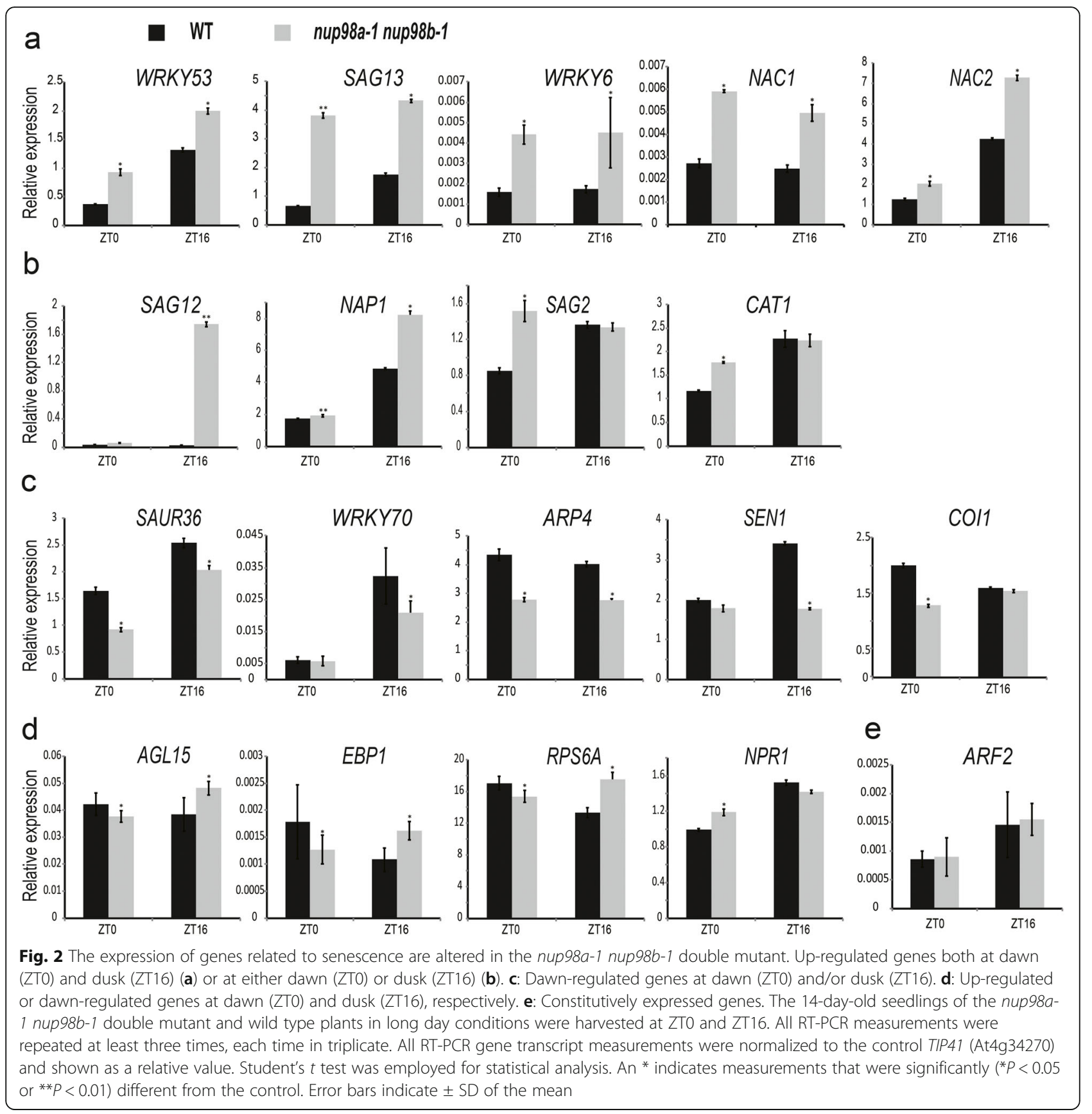

only in the early steps in chloroplasts [17], such as GWD1 (encoding $\alpha$-GLUCAN WATER, DIKINASE1; or STARCH EXCESS1, SEX1), $\beta$-BAM1 (encoding $\beta$ AMYLASE1), BAM3, BAM5, BAM6, BAM7, BAM8, SEX4, and LSF1 (encoding LIKE SEX FOUR1), but also in the later steps, such as LDA (encoding LIMITDEXTRINASE), $A M Y 1$ (encoding $\alpha$-AMYLASE) and $A M Y 2$, DPE1 (encoding DISPROPORTIONING ENZYME) and DPE2, PHS1 (encoding $\alpha$-GLUCAN PHOSPHORYLASE) and PHS2. Time-dependent lowexpression of these genes suggests that they are under circadian control and Nup98 itself is involved in circadian regulation in Arabidopsis [12]. These genes function at different steps of starch degradation [17]. GWD1 is $\alpha$-glucan water dikinase, which phosphorylates glucosyl residues of amylopectin at the $\mathrm{C}-6$ position. BAMs are a family of $\beta$-amylases breaking down the $\alpha$-1,4linked glucose chains. LSF1 and SEX4 release the phosphate bound at C- 6 and C-3 of glucosyl residues. Both ISA (ISOAMYLASE 3) and LDA (LIMIT-DEXTRINASE) hydrolyze $\alpha-1,6$ branch points but have different substrates. AMYs act on $\alpha$-1,4-linkages releasing linear 


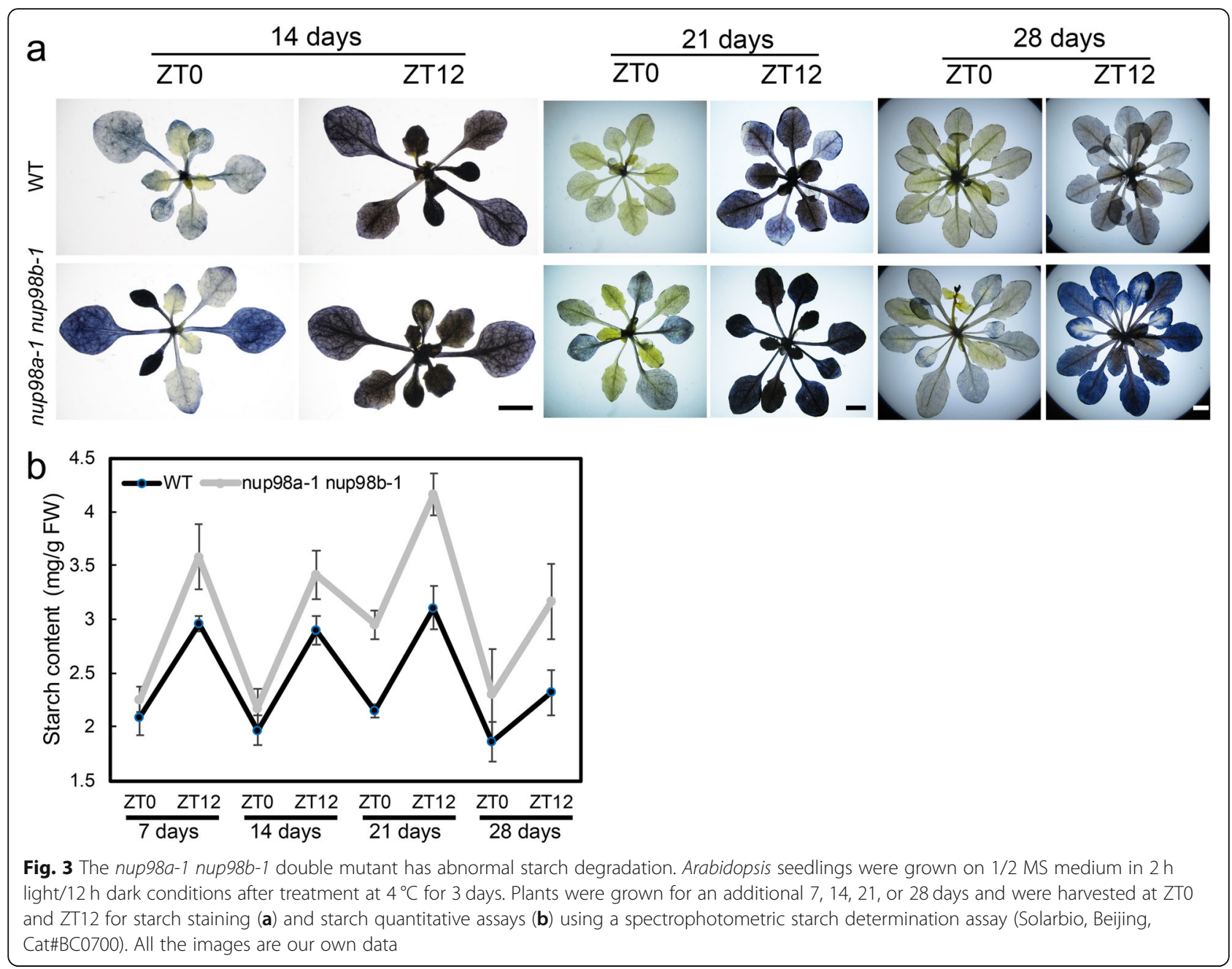

$\alpha-1,4$-linked oligosaccharides and branching $\alpha-1,4-$ and $\alpha$-1,6-linked oligosaccharides. DPEs (DISPROPORTIONATING ENZYMEs) transfer glucose/ $\alpha$-1,4-linked glucan moiety from a donor glucan to an acceptor, releasing the non-reducing end of glucose/glucan moiety. PHSs ( $\alpha$--GLUCAN PHOSPHORYLASEs) act on the non-reducing end of $\alpha$-1,4-linked glucose. Lower abundance of these genes resulted in a starch-excess phenotype in the nup98a-1 nup98b-1 double mutant (Fig. 3) as sex4 mutants [17]. The mRNA abundance of some other genes involved in starch metabolism, including GWD2, GWD3, ISA3 (ISOAMYLASE3) and AMY3, was not significantly affected, suggesting the effect of Nup $98 a / b$ on starch metabolism is limited to some specific enzymes.

We also measured the mRNA abundance of genes related to photosynthesis and sugar metabolism by RT-qPCR in the nup $98 a-1$ nup $98 b-1$ double mutant and wild type plants (Supplementary Figure 8). In terms of photosynthesisrelated genes, the decrease of mRNA abundance of $L H C A$ (PHOTOSYSTEM I LIGHT HARVESTING COMPLEX
GENE A) and LHCB (PHOTOSYSTEM I LIGHT HARVESTING COMPLEX GENE B) was observed in the nup 98a-1 nup 98b-1 double mutant at different time points, e.g., $L H C A 1 / 2$ and LHCB1.1 at ZT0 and LHCA1 and LHCB1.4 genes at ZT16. We also observed a decrease in mRNA abundances of KIN10 and KIN11 in the double mutant when compared to wild type plants, which is expected since both of genes are sugar signaling genes. Both these genes are involved in delaying plant senescence [21, 26, 27], such that their reduced transcript abundance may be associated with earlier senescence (Supplementary Figure 6). We also observed slightly increased mRNA abundance of HXK1 at dusk (ZT16) that may contribute to earlier senescence via the cytokinin signaling pathway [24]. Unexpectedly, mRNA abundance of TPS1, a senescence activator [20], was reduced in the nup98a-1 nup $98 b-1$ double mutant compared with wild type plants, suggesting that T6P (trehalose-6phosphate) was not related to the observed senescence. The results indicate that starch synthesis and sugar signalling are impaired in the double mutant. 


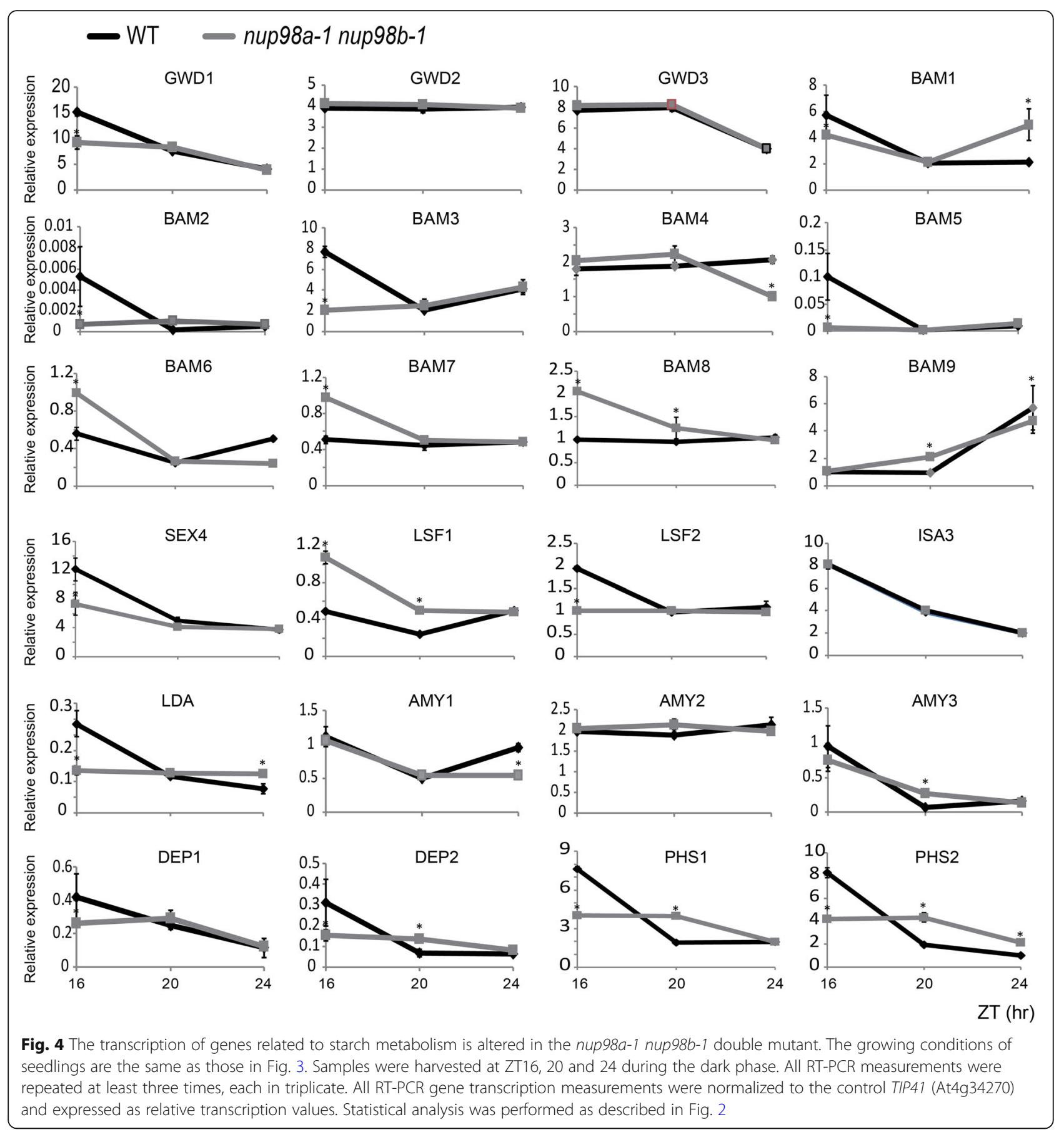

Exogenous sugar rescues the early senescence phenotype in the nup98a-1 nup98b-1 double mutant

Our results suggested that the carbon or energy supply was impaired in nup $98 a-1$ nup $986-1$ double mutant plants. We tested the idea by supplying exogenous carbon in the form of sucrose in the growing medium to see if the early senescence phenotype could be rescued. Our results showed that sucrose and MS basal nutrients supported good plant growth even though it was weaker than wild type plants (Fig. 5). Both plants completed their life cycles on medium supplemented with sucrose and basal nutrients. Next, we allowed double mutant and control plants to grow in MS medium until inflorescence emergence and then transferred them to soil. As expected, the double mutant grew as well as wild type plants on MS medium before transplanting (Supplementary Figure 9). However, after transferring to soil, senescence symptoms quickly appeared at day 6 on mutant plant leaves, and the mutant plants wilted at day 30 (Supplementary Figure 9). Wild type plants and the 


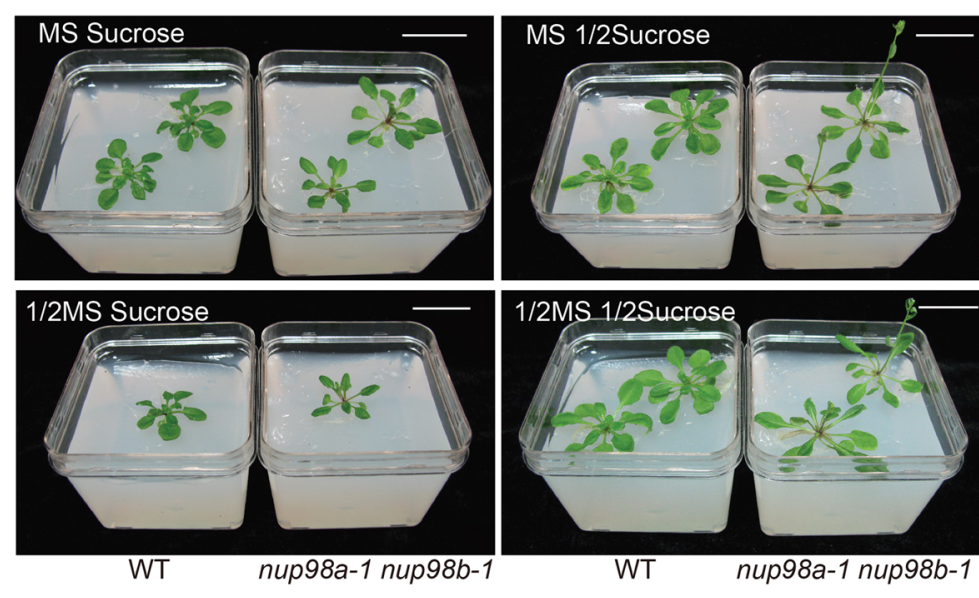

The 28 day-old plants
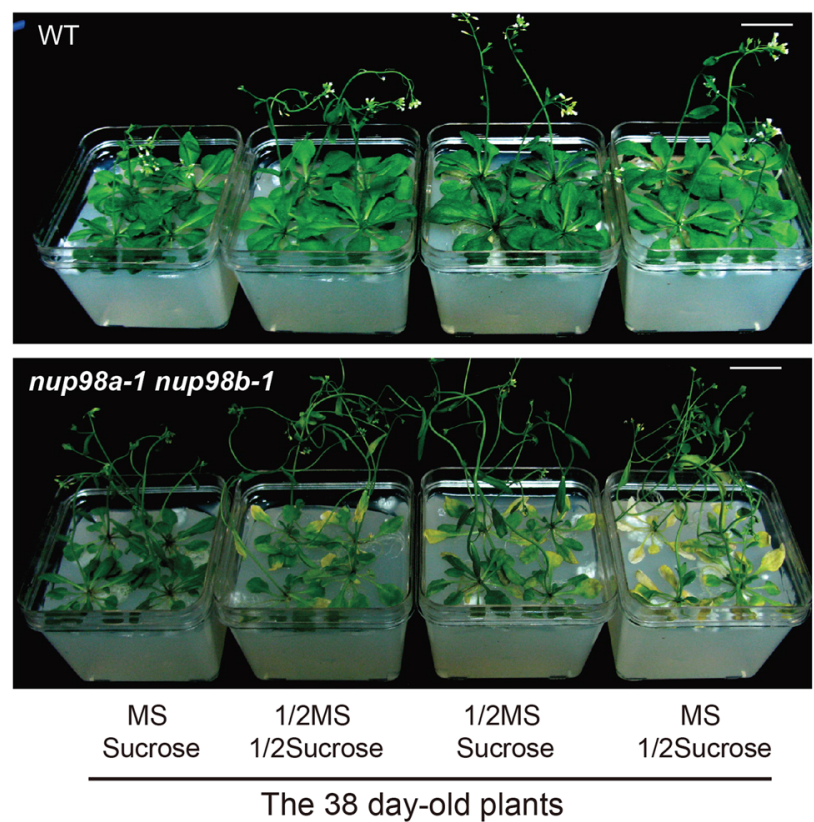
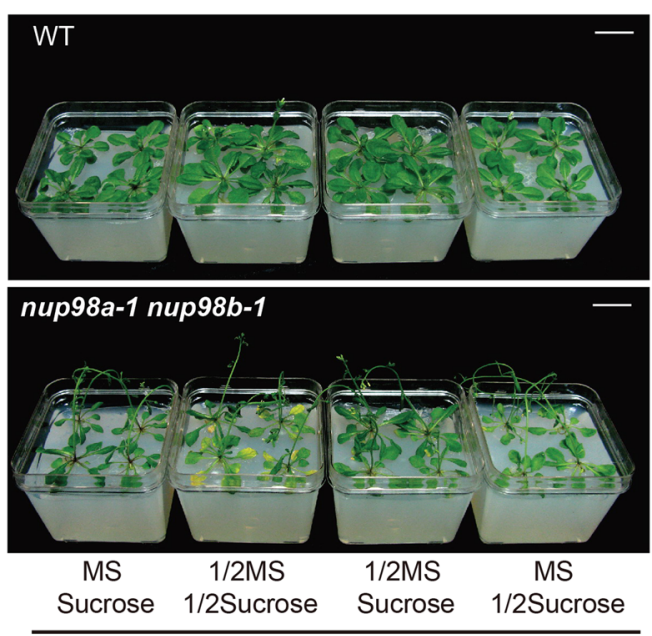

The 33 day-old plants
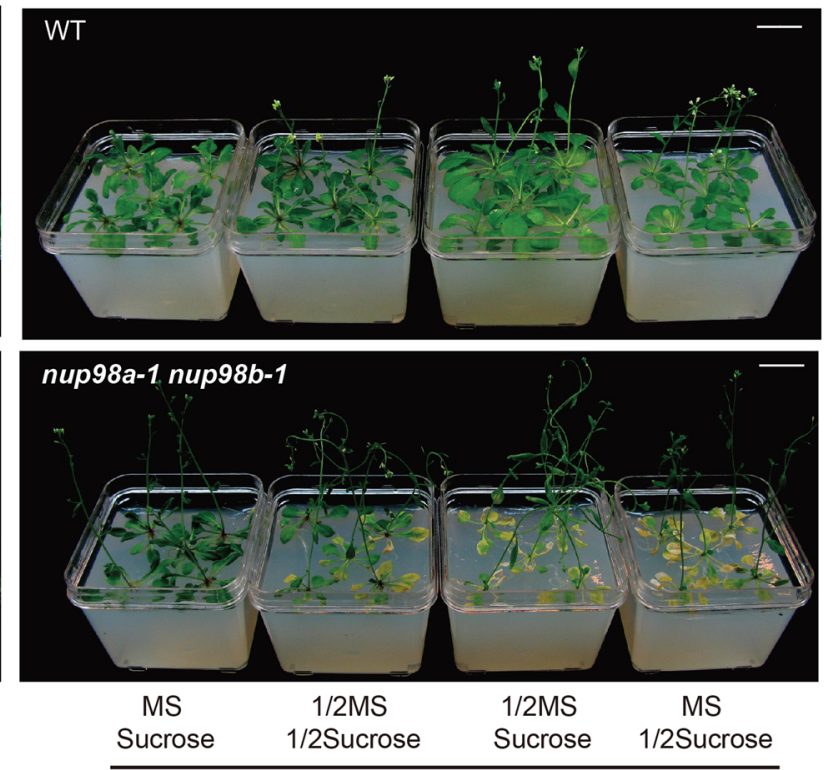

The 42 day-old plants

Fig. 5 Increased nutrients and sucrose delays senescence in nup98a-1 nup98b-1 double mutants. The growing conditions of seedlings are same as those in Fig. 3. 1/2MS indicates 1/2 strength of MS. 1/2 sucrose indicates 1.5\% sucrose. After stratification, plants were grown under long day conditions. Increases in basal nutrients and sucrose both delayed senescence in the nup98a-1 nup98b-1 double mutant plants. Bars $=5 \mathrm{~cm}$. All the images are our own data

nup 98 double mutant would not survive as plants grown on agarose medium without any supplements (Supplementary Figure 10).

To rule out the possible effect of soil on the senescence phenotypes observed above, we tested whether exogenous macro and micronutrients would complement the phenotypes observed in the double mutant by continuously growing plants on medium at different strengths of sucrose and macro- and micro-nutrients (Supplementary Figure 10). To our surprise, not only did sucrose suppress the early senescence phenotype in the double mutant but also macro- and micro-nutrients in the presence of sucrose. The lower strength basal nutrients ( $1 / 2 \mathrm{MS})$ enhanced the lower sucrose (1.5\%) effect on suppressing senescence, suggesting that both energy supply and basal nutrient metabolism are impaired in the nup 98 a-1 nup $98 b-1$ double mutant.

Starvation and senescence marker genes express highly in the nup98a-1 nup986-1 double mutant

Results above imply that the nup $98 a-1$ nup $98 b-1$ double mutant may suffer from sugar starvation and senescence. Therefore, we asked if markers genes of starvation or senescence expressed highly in the double mutant. 
DORMANCY-ASSOCIATED PROTEIN-LIKE 1 (DRM1/ DYL1, At1g28330) and DARK INDUCIBLE 6 (DIN6, At3g47340) [38, 39] are two well-studied sugar starvation marker genes, whereas SAG12 (At5g45890) and WRKY53 (At4g23810) are well-characterized senescence markers [40-42]. Autophagy is an important event occurring during sugar starvation and senescence [38, 43, 44], and AUTOPHAGY8a (ATG8a, At4g21980) and ATG8e (At2g45170) are two typical molecular indicators for autophagy in plants [45]. Therefore, we investigated expression changes of these genes in the nup $98 a-1$ nup $98 b-1$ double mutant compared with those in wild type plants, and the results showed that they had different changes in a time- and developmental-dependent mode (Fig. 6). In the double mutant, DRM1 began significantly higher expression at ZT0, but lower at ZT12 at a very early stage (day 5 after germination) (Fig. 6a). $D I N$ is a light-repressed and dark-induced gene [46], and its high level of expression at ZT0 in the double mutant became obvious at day 15, but at ZT12 higher abundancy appeared earlier from day 10 (Fig. 6a). Compared with wild type plants, the senescence marker WARKY53 in the double mutant expressed higher at the early stage when DRM1 expression was in disorder (day 5) (Fig. 6b). SAG12 is a developmentally-controlled indicator for the later stage of senescence $[47,48]$. We found that there was not much difference in SAG12 expression at the early stage (day 5 ) between the double mutant and wild

a, Sugar starvation gene markers
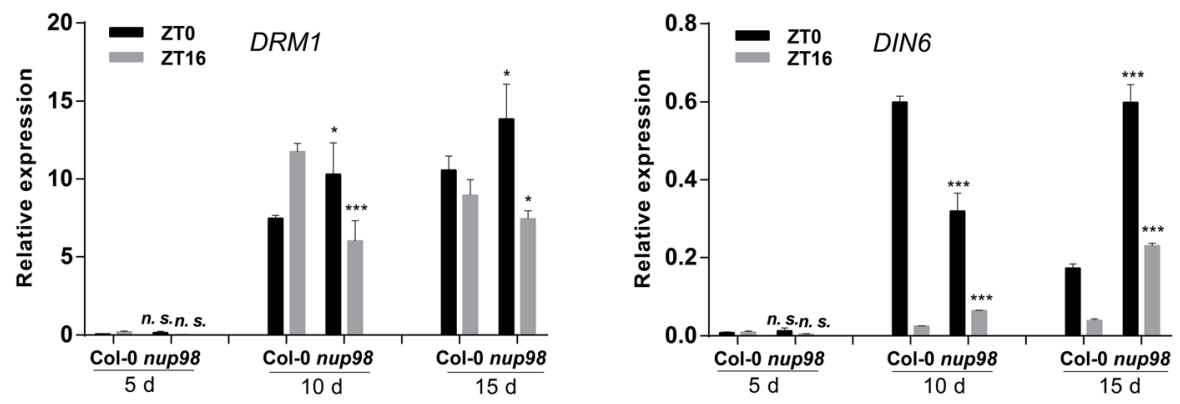

b, Senecence gene markers
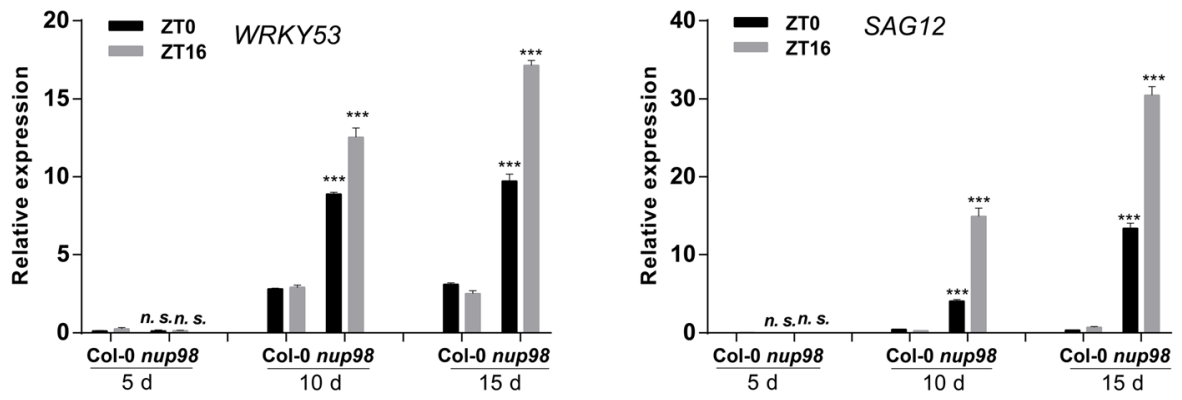

C, Autophagy gene markers
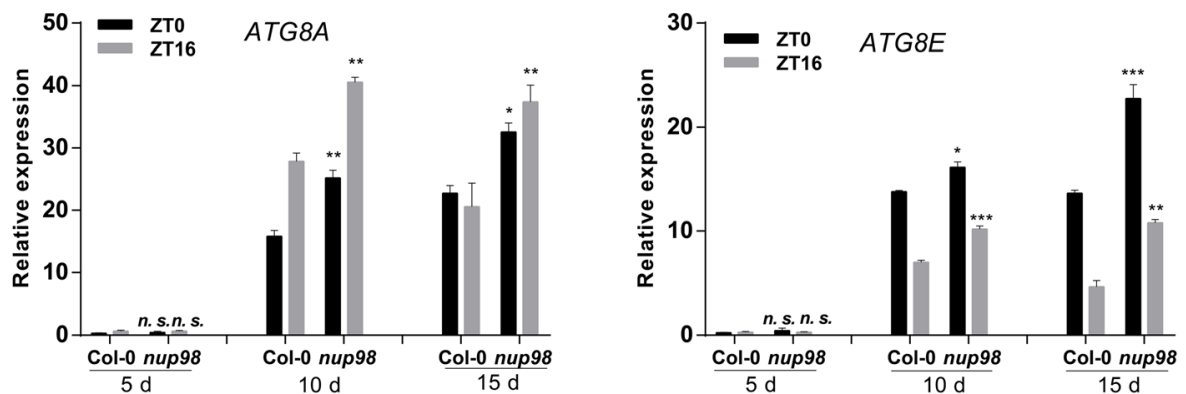

Fig. 6 Marker genes of starvation and senescence show altered transcript accumulation in the nup98a-1, nup98b-1 double mutant. a: Sugar starvation genes. b: Senescence genes. c: Autophagy genes. The growth conditions of seedlings are the same as those in Fig. 3. After low temperature treatment for 3 days, the seedlings were grown for 7 days under long day conditions, then transplanted into soil. Samples were harvested on day 5, 10, and 15 at both ZT0 and ZT16. Triplicate QPCR measurements were repeated at least three times, normalized to the reference gene TIP41 (At4g34270) and expressed as relative transcript accumulation values. Statistical analysis as described in Fig. 2 
type plants. However, SAG12 had a higher transcript level in the double mutant at both ZT0 and ZT12 from day 10 (Fig. 6b). The two markers of autophagy, ATG8a and $A T G 8 e$, also had higher abundancy of mRNA in most samples of the double mutant from day 10. Taken together, our results showed that the nup $98 a-1$ nup $98 b$ 1 double mutant presented signs of energy starvation, at least at the molecular level, during early developmental stages when plants did not display visible senescence phenotypes. These expression changes had circadian and developmental characters. A previous report shows that different sugars (such as sucrose, glucose, and fructose) have different effects on the regulation of senescence [39]. Therefore, the observance of early and high expression of the marker genes was in accord with senescence characteristics of the nup $98 a-1$ nup $98 b-1$ double mutant.

\section{Nup98 proteins mainly localize to both the nuclear membrane and nucleoplasm}

Nup98 is one of the mobile and peripheral FG (Phe-Gly domain) nucleoporins which is located at both the nuclear and cytoplasmic sides of the NPC central channel $[4,5]$. Arabidopsis Nup98a (also known as DRA2) is also found in different subcellular compartments [6]. We constructed transgenic Arabidopsis plants expressing 35S::GFP:Nup98a and 35S::GFP:Nup98b and analyzed the subcellular localization of both fusion proteins. Not surprisingly, both proteins were distributed in the cytoplasm, in the nucleoplasm and at the nuclear periphery (Fig. 7). We observed no significant difference in the subcellular distribution of the two proteins and this is consistent with our observations of their genetic redundancy. In conclusion, our combined results demonstrate that Nup98b proteins localize in both the nucleus and cytoplasm as do Nup98a proteins [6] and their homologs in other organisms $[4,5]$.

\section{Discussion}

Senescence is a process that is integral to growth and development during the plant life cycle, eventually leading to cell and tissue disintegration and death. In such a physiological process, various basal nutrients are redistributed from senescing organs, such as leaves, to reproductive organs and seeds [13]. However, premature senescence is likely to lead to organ failure or even whole plant death [49]. Fine tuning senescence processes could benefit plants by avoiding the deleterious effect of abiotic stresses and thereby lead to an optimal reproductive outcome. A number of factors, including hormones, developmental age, abiotic stress and light conditions, participate in the regulation of plant senescence $[21,22,50-59]$. While these factors play important and clear roles in plant senescence, the role of sugar is unclear as different research groups have published contradictory results $[19-21,60]$. The NPC is an important gatekeeper for both macromolecular transportation between the nucleus and cytoplasm and gene transcription and, therefore, it plays an important role in many different developmental processes in plants [28-32]. Our study provides some novel insights into plant senescence research as we found that the NPC participated in
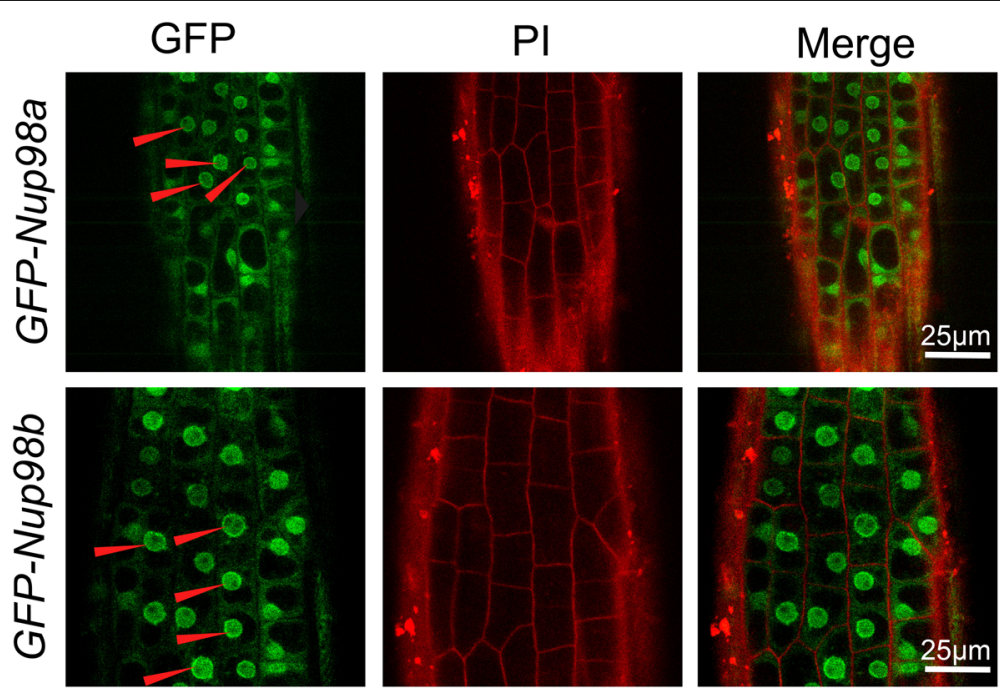

Fig. 7 Nup98a and Nup98b proteins are localized to the nuclear membrane and in the nucleoplasm. The green fluorescent protein (GFP) was fused to either Nup98a or Nup98b to generate N-terminal translational fusional proteins, GFP-Nup98a and GFP-Nup98b. Integration of gene constructs driven by the CaMV 355 promoter gave rise to stably-transformed Arabidopsis thaliana plants. Arrow heads indicate that both GFPNup98a and GFP-Nup98b were enriched near the nuclear periphery when compared to the cytoplasm. PI (propidium iodide) was used for cell wall staining. All the images are our own data 
senescence regulation. Our investigation confirmed that Nup98 genes are involved in starch degradation, conferring senescence initiation in Arabidopsis.

Nup $98 a$ and Nup $98 b$ function redundantly in senescence regulation in Arabidopsis, because the nup 98 single mutants have no obvious senescence phenotypes (Fig. 1), even though a previous report showing longer hypocotyls in the nup 98 a mutant [6]. Similar protein sequences (Figure S1) and subcellular-localization between the Nup98a and Nup98b proteins (Fig. 7) support this hypothesis. Such a senescence phenotype is likely to be specific to mutation of Nup98 genes, because other nucleoporin mutants detected do not have similar phenotypes (Figure S4). However, it is important to check more mutants of other nucleoporins to elucidate the specificity of Nup 98 functions on senescence regulation. Molecular data support that the Nup98 genes regulate senescence through multiple pathways, including ethylene, salicylic acid, ABA, cytokinin, and stress, because the marker genes in these pathways, such as SAG12, NAP1, WRAY53, WRKY6, WRKY70, NAC1, NAC2 and $H X K 1$, have significant changes in gene expression (Fig. 2). Additionally, these genes may play a role in a temporal (circadian) manner as their significant expression changes are only at a specific time, at either dawn or dusk (Fig. 2). We recently reported that the expression level of ELF3 gene and other clock genes significantly reduced in the nup98a-1 nup98b-1 double mutant compared with that in wild type plants [12]. Therefore, circadian patterns may be involved in the senescence network regulated by the Nup98-encoding genes.

Sugar is confirmed as an important signal in the control of plant growth and development [61]. Many important genes in sugar signaling pathways (TOR, KIN10, KIN11, TPS1, SnRK1) (Figure S7) and carbon starvation (DRM1 and DIN6) (Fig. 6) were all mis-regulated in nup 98 a-1 nup $98 b-1$ double mutant plants. Based on the data presented, sugar availability is likely hindered in the double mutant, suggesting that this might be the main reason for the initiation of senescence (Fig. 5).

In plants, sugar is derived from photosynthesis and stored firstly as starch. Starch accumulated in the chloroplast during the day is broken down into monosaccharides at night $[17,18]$. Our evidence showed the nup 98 a-1 nup $98 b-1$ double mutant accumulated much higher levels of starch, which was unlikely due to higher photosynthetic efficiency (Figure S7) but impaired starch degradation (Fig.3). This hypothesis was supported by 1 ) lower transcription levels of genes related to photosynthesis (LHCA1, LHCA2, LHCB1.1 and LHCB1.4), which would lead to reduced starch synthesis in the double mutant (Figure S7); 2) lower transcript accumulation of starch degradation genes (Fig. 4) controlling many steps in starch degradation (Figure S6) [17] that would lead to starch accumulation in the nup $98 a-1$, nup $98 b-1$ double mutant. Impairment of starch degradation in the double mutant could lead firstly to sugar starvation, subsequently to leaf senescence, and finally to whole plant death.

Beyond this, Nup98a and Nup98b may also have functions in nutrient metabolism as the concentration of basal nutrients in the growth medium had a significant impact on the growth and senescence in nup98a-1, nup $98 b-1$ double mutants (Fig. 5). In this case, components (sugar or MS) in growth media should be taken into consideration, especially in future senescence studies.

Previous experiments show that the circadian clock regulates starch metabolism in plants [62, 63]. ELF3 positively regulates starch accumulation, and degradation of starch was significantly slower in elf3 mutant plants than in the corresponding wild type plants [64]. Therefore, ELF3 and other clock evening genes, such as ELF4 and LUX ARRHYTHMO, also affect leaf senescence $[65,66]$. Many genes studied here showed timedependent differences in gene expression (Figs. 2, 4 and 6). We previously showed that the expression of many clock genes significantly changed in the nup98a-1, nup 98b-1 double mutant [12]. Taken together, clock genes might participate in signalling pathways of Nup 98a/Nup98b-regulating starch degradation and senescence in Arabidopsis.

We recently reported that the nup98a-1, nup $98 b-1$, ft10 triple mutant displays the same late-flowering character as the $f t-10$ mutant but it also maintains the early senescence phenotypes of the nup98a-1, nup $98 b-1$ double mutant [12], suggesting that Nup98a and Nup $98 b$ proteins are involved in the regulation of flowering and senescence processes in unrelated or independent pathways.

It is clear that the function of Nup98 in senescence regulation is indirect. Mutation of Nup 98 genes may lead sequentially to dysfunction of circadian pathways, hinderance of starch degradation, sugar starvation, leaf senescence, and plant death. It would be interesting to determine how Nup 98 controls the function of genes related to starch degradation mediated by circadian clockrelated mechanisms.

It is should be pointed that even though Nup98a and Nup $98 b$ function redundantly in the regulation of flowering and senescence, they may play independent and specific functions in other developmental processes, because the nup 98 a single mutant (dra2) displays shade avoidance [6].

\section{Conclusion}

NPC has multiple functions in plant development. To the best of our knowledge, our study presents the first 
report of NPC functions in regulating plant senescence. This result is promising and implies a novel function of NPC in bridging the gap between starch metabolism and senescence control. That is, Nup98a and Nup98b proteins overlap in their control of starch degradation conferring senescence regulation in Arabidopsis.

\section{Methods}

Plant materials and growth conditions

Seeds of the T-DNA insertion mutants of nup 98 a (SALK_080083, SALK_090744, SALK_023493, SALK_ 103803, and SALK_015016) and nup98b (CS803848 and GABI_288A08) were ordered from ABRC and GABI TDNA mutant center, respectively. Homozygous screening was according to the protocol provided by SALK (http://signal.salk.edu/). All mutants were identified by Dr. Long Xiao. Except where indicated in the text, Arabidopsis thaliana Columbia wild type plants and their derived mutants were grown under long day conditions $\left(16 \mathrm{~h} / 8 \mathrm{~h}\right.$, light/dark) with $100 \mu \mathrm{mol} \mathrm{m}{ }^{-2} \mathrm{~s}^{-1}$ fluorescent lighting. Plants were grown in soil in $10 \mathrm{~cm}$ pots or on medium in petri dishes containing different strengths of sucrose and MS (Murashige \& Skoog) basal medium (Sigma, \#M5524), which contains macronutrients and micronutrients of the original classic formulation $\left(\mathrm{NH}_{4} \mathrm{NO}_{3}, 1650.0 \mathrm{mg} \cdot \mathrm{L}^{-1} ; \mathrm{KNO}_{3}, 1900.0 \mathrm{mg} \cdot \mathrm{L}^{-1} ; \mathrm{CaCl}_{2}\right.$, $332.2 \mathrm{mg} \cdot \mathrm{L}^{-1} ; \quad \mathrm{MgSO}_{4} \cdot 7 \mathrm{H}_{2} \mathrm{O}, \quad 180.7 \mathrm{mg} \cdot \mathrm{L}^{-1} ; \quad \mathrm{KH}_{2} \mathrm{PO}_{4}$, $170.0 \mathrm{mg} \cdot \mathrm{L}^{-1} ; \mathrm{FeSO}_{4} \cdot 7 \mathrm{H}_{2} \mathrm{O}, 27.8 \mathrm{mg} \cdot \mathrm{L}^{-1} ; \mathrm{H}_{3} \mathrm{BO}_{3}, 6.2$ $\mathrm{mg} \cdot \mathrm{L}^{-1} ; \mathrm{MnSO}_{4} \cdot \mathrm{H}_{2} \mathrm{O}, 16.9 \mathrm{mg} \cdot \mathrm{L}^{-1} ; \mathrm{CoCl}_{2} \cdot 6 \mathrm{H}_{2} \mathrm{O}, 0.025$ $\mathrm{mg} \cdot \mathrm{L}^{-1} ; \mathrm{ZnSO}_{4} \cdot 7 \mathrm{H}_{2} \mathrm{O}, 8.6 \mathrm{mg} \cdot \mathrm{L}^{-1} ; \mathrm{Na}_{2} \mathrm{MoO}_{4} \cdot 2 \mathrm{H}_{2} \mathrm{O}, 0.25$ $\mathrm{mg} \cdot \mathrm{L}^{-1}$; $\mathrm{KI}, 0.83 \mathrm{mg} \cdot \mathrm{L}^{-1}$; $\mathrm{CuSO}_{4} \cdot 6 \mathrm{H}_{2} \mathrm{O}, 0.025 \mathrm{mg} \cdot \mathrm{L}^{-1}$; $\mathrm{Na}_{2}$ EDTA. $2 \mathrm{H}_{2} \mathrm{O}, 37.26 \mathrm{mg} \cdot \mathrm{L}^{-1}$; Glycine (free base), 2 $\mathrm{mg} \cdot \mathrm{L}^{-1}$; myo-Inositol, $100 \mathrm{mg} \cdot \mathrm{L}^{-1}$; Nicotinic acid (free acid), $0.5 \mathrm{mg} \cdot \mathrm{L}^{-1}$; Pyridoxine $\cdot \mathrm{HCl}, 0.5 \mathrm{mg} \cdot \mathrm{L}^{-1}$; Thiamine. $\mathrm{HCl}, 0.1 \mathrm{mg} \cdot \mathrm{L}^{-1}$ ) [67].

\section{Gene and promoter cloning and plasmid construction}

Standard GATEWAY (Invitrogen) methods were employed for gene cloning and plasmid construction. Most vectors were developed by our lab [68]. The fulllength of Nup98a and Nup98b open reading frames was PCR-amplified with specific primers (Table S1), and then cloned into Fu30 [68] containing an N-terminal GFP marker. The gene entry vectors (Fu30-GFP:Nup $98 a$ or Fu30-GFP:Nup98b), the $35 S$ promoter entry vector (Fu76-35S) and the binary vector (Fu39-2) [68] were applied to LR reaction (Invitrogen). Both Fu39-2-35S:GFP: Nup98a and Fu39-2-35S:GFP:Nup98b binary vectors were applied to transform $A$. thaliana using the floral dipping method [69]. Homozygous transgenic lines were used for molecular characterization and phenotype analysis.
Semi-quantitative PCR, quantitative real time RT-PCR, and subcellular localization

Whole seedlings were harvested at ZT0 (the time point of light on) and ZT16 (the time point of light off) at day 14 after germination. RNA preparation, cDNA synthesis and both quantitative real-time and semi-quantitative RT-PCRs were carried out following Xiao et al. [70], except for the use of At4g34270 as a reference gene in triplicate [71, 72]. All gene accession numbers and relevant primer sequences are listed in Table S1. GFP fluorescent signals were visualized and captured by confocal microscopy, while propidium iodide (PI) was employed for cell wall staining [68].

\section{Starch staining and quantification analyses}

Starch in leaves of 14-21-, and 28-day-old wild type plants and mutant Arabidopsis plants was stained with iodine [73]. Samples were harvested at ZT0 (the time point of light on) and ZT12 (the time point of light off) in a $12 \mathrm{~h}$ light $/ 2 \mathrm{~h}$ dark cycle. Representative plants were used to build up figures. Starch quantitative analysis was carried out according to the instructions of the Starch Determination Kit (Solarbio, Beijing, Cat\#BC0700) on a spectrophotometer (RAYLEIGH, VIS$7220 \mathrm{~N}$; Beijing Beifen-Ruili Analytical Instruments (Group) Co., Ltd) using standard curves which were generated with different concentration of glucose $(0.2,0.1$, $0.05,0.025,0.0125,0.00625,0.003125,0.00156 \mathrm{mg} / \mathrm{mL})$.

\section{Statistical analysis}

Each experiment had at least three biological replicates and similar results were obtained for each replicate. For figures, one representative plant was selected and photographed. The SPSS software package was used to identify significant difference probability $(P)$ levels for all data $\left({ }^{*} P<0.05,{ }^{* *} P<0.01\right.$, and ${ }^{* * *} P<0.001$, compared to controls). Error bars represent \pm SD of the mean.

\section{Supplementary information}

Supplementary information accompanies this paper at https://doi.org/10. 1186/s12870-020-02494-1.

Additional file 1: Supplementary Table 1. Primers used in this study. Additional file 2: Figure S1. Protein domains in Nup98a and Nup98b of Arabidopsis thaliana.

Additional file 3: Figure S2. The original photograph of the gel in Fig. $1 \mathrm{~b}$.

Additional file 4: Figure S3. The nup98a1, nup98b1 double mutant showed pleiotropic phenotypes in various organs.

Additional file 5: Figure S4. The nup98a1, nup98b1 double mutant showed a senescent and sterile phenotype.

Additional file 6: Figure S5. Senescent phenotypes were specific to the nup98a1, nup9861 double mutant compared with mutants of other nucleoporins. 
Additional file 7: Figure S6. Molecular network of senescence initiation in plants.

Additional file 8: Figure S7. A simplefied pathway of starch degradation in chloroplasts.

Additional file 9: Figure S8. Expression analysis of genes related to photosynthesis and sugar metabolism in the nup98a1, nup9861 double mutant.

Additional file 10: Figure S9. Exogenous sucrose reduces early senescence in the nup98a1, nup9861 double mutant plants.

Additional file 11: Figure S10. Phenotype analysis of the nup98a1, nup98b1 double mutant compared with WT grown on different mediums.

\section{Abbreviations}

NPC: Nuclear pore complex; Nup: Nucleoporin; CO: CONSTANS; ABA: Abscisic acid; ABI: ABA INSENSITIVE; ACS: Aminocyclopropane-1-carboxylate (ACC) synthase; AGL15: AGAMOUS-LIKE 15; AHK: Arabidopsis histidine kinase; AHP: Arabidopsis thaliana histidine phosphotransfer proteins; AMY3: Alphaamylase-like 3; APD: Autoproteolytic domain; APIP12: AVRPIZ-T INTERACTING PROTEIN12; ARP4: ACTIN-RELATED PROTEIN4; ARF: Auxin response factor; ARR: Arabidopsis response regulator; $\mathrm{C} 2 \mathrm{H} 4$ : Ethylene; ATG8: Autophagy8; BAM: Beta-amylase; CAT: Catalase; CK: Cytokinin; COI1: Coronatine insensitive1; CWINV: Cell wall invertase; DIN6: Dark inducible6; DRM1/ DYL1: Dormancy-associated protein-like1; DPE: Disproportionating enzyme; DRA2: DRACULA2; EBP1: Erbb-3 binding protein; EEL: Enhanced em level; EIN: ETHYLENE INSENSITIVE; ELF: EARLY FLOWERING; ETR: ETHYLENE RESPONSIVE; FG: Phe-Gly domain; G6P: 6-phosphoric acid glucose; GFP: GREEN FLUORESCENT PROTEIN; GLK: GOLDEN 2-LIKE TRANSCRIPTION FACTOR; GWD1: a-glucan water: dikinase; HXK1: Hexokinase1; ISA3: Isoamylase3; KIN: PROTEIN KINASE; LDA: Limit-dextrinase; LHCA: PHOTOSYSTEM I LIGHT HARVESTING COMPLEX GENE A; LHCB: PHOTOSYSTEM I LIGHT HARVESTING COMPLEX GENE B; LSF1: Like sex four1; N: Nitrogen; NAC: NAM, ATAF, AND CUC; NAP: NAC-LIKE PROTEIN; NPR1: NONEXPRESSER OF PR GENES 1; NYC: NONYELLOW COLORING; MOS7: MODIFIER OF SNC1,7; ORE: ORESARA; PHS: a-glucan phosphorylase; PHYB: PHYTOCHROME B; PIF: PHYTOCHROME INTERACTING FACTOR; PYR/PYL/RCAR: PYRABACTIN RESISTANCE/PYR1-LIKE ORREGULATORY COMPONENT OF ABA RECEPTOR; RPS6A: RIBOSOMAL PROTEIN S6A; RT-qPCR: Real time quantitative PCR; SAG: SENESCENCE-ASSOCIATED GENE; SAS: SHADE AVOIDANCE SYNDROME; SAUR: SMALL AUXIN UPREGULATED; SAUR36: SMALL AUXIN UPREGULATED; SEX4: STARCH EXCESS 4; SGR: STAYGREEN; T6P: Trehalose-6-phosphate; TOR: TARGET OF RAPAMYCIN; Tpr: RANSLOCATED PROMOTER REGION; TPS1: Trehalose-6-phosphate synthase1; WRKY: WRKY DNA-BINDING PROTEIN; ZT: Zeitgeber

\section{Acknowledgments}

We thank all friends and colleagues who helped us. Thanks to Prof. Jeremy Timmis at The University of Adelaide for his improving English of the MS.

\section{Authors' contributions}

Conceptualization, Y-FF and X-MZ; Methodology, LX and Y-FF; Investigation, $L X, S J, P H, F C, Z C, Y M, X-X W, C L$, and LL; Formal analysis, LX, FC, XW, and QC; Visualization, LX and Y-FF; Writing -Original Draft, Y-FF, and QC Writing Review \& Editing, IS and Y-FF; Funding Acquisition, Y-FF and X-MZ; Project administration, Y-FF and X-MZ; Resources, X-M Z, YM, X-XW, and QC; Supervision, $Y$-FF. All Authors read and approved the manuscript.

\section{Funding}

This research was supported by National Natural Science Foundation of China (Grant No. 31571411 and 31370324), CAAS-Innovation Team Project, Australian Research Council Future Fellowship (FT130100525) and the Basal Research Fund of CAAS (Y2017CG25). The funders were not involved in the experimental design of the study, data collection, analysis and interpretation, and in writing the manuscript.

\section{Availability of data and materials}

All data generated or analyzed during this study are included in this published article and its supplementary information files.

\section{Ethics approval and consent to participate}

Not applicable.

\section{Consent for publication}

Not applicable.

\section{Competing interests}

The authors declare that they have no competing interests.

\section{Author details}

${ }^{1}$ Key Laboratory of Soybean Biology, Ministry of Education/College of Agriculture, Northeast Agricultural University, Harbin 150030, China. ${ }^{2} \mathrm{MOA}$ Key Lab of Soybean Biology (Beijing), National Key Facility of Crop Gene Resource and Genetic Improvement, Institute of Crop Sciences, Chinese Academy of Agricultural Sciences, Nandajie 12, Zhongguancun, Haidian District, Beijing 100081, China. ${ }^{3}$ Key Laboratory of Plant Stress Biology, State Key Laboratory of Cotton Biology, School of Life Sciences, Henan University, Kaifeng 475004, China. ${ }^{4}$ College of Life Sciences, Capital Normal University, Beijing 100048, China. ${ }^{5}$ School of Biological Sciences, School of Agriculture, Food and Wine, The University of Adelaide, Adelaide, South Australia 5005, Australia.

Received: 19 December 2019 Accepted: 15 June 2020

Published online: 26 June 2020

\section{References}

1. Weis K. Regulating access to the genome: nucleocytoplasmic transport throughout the cell cycle. Cell. 2003;112(4):441-51.

2. Strambio-De-Castillia C, Niepel M, Rout MP. The nuclear pore complex: bridging nuclear transport and gene regulation. Nat Rev Mol Cell Biol. 2010; 11(7):490-501.

3. Tamura K, Fukao Y, Iwamoto M, Haraguchi T, Hara-Nishimura I. Identification and characterization of nuclear pore complex components in Arabidopsis thaliana. Plant Cell. 2010;22(12):4084-97.

4. Capitanio JS, Montpetit B, Wozniak RW. Nucleoplasmic Nup98 controls gene expression by regulating a DExH/D-box protein. Nucleus. 2018;9(1):1-8.

5. Franks TM, McCloskey A, Shokirev MN, Benner C, Rathore A, Hetzer MW. Nup98 recruits the Wdr82-Set1A/COMPASS complex to promoters to regulate $\mathrm{H} 3 \mathrm{~K} 4$ trimethylation in hematopoietic progenitor cells. Genes Dev. 2017;31(22):2222-34

6. Gallemi M, Galstyan A, Paulisic S, Then C, Ferrandez-Ayela A, Lorenzo-Orts L, Roig-Villanova I, Wang X, Micol JL, Ponce MR, et al. DRACULA2 is a dynamic nucleoporin with a role in regulating the shade avoidance syndrome in Arabidopsis. Development. 2016;143(9):1623-31.

7. Light WH, Brickner JH. Nuclear pore proteins regulate chromatin structure and transcriptional memory by a conserved mechanism. Nucleus. 2013;4(5): 357-60.

8. Cross MK, Powers MA. Nup98 regulates bipolar spindle assembly through association with microtubules and opposition of MCAK. Mol Biol Cell. 2011; 22(5):661-72.

9. Parry G. Components of the Arabidopsis nuclear pore complex play multiple diverse roles in control of plant growth. J Exp Bot. 2014;65(20): 6057-67.

10. Tang MZ, Ning YS, Shu XL, Dong B, Zhang HY, Wu DX, Wang H, Wang GL, Zhou B. The Nup98 homolog APIP12 targeted by the effector AvrPiz-t is involved in rice basal resistance against Magnaporthe oryzae. Rice. 2017; 10:5

11. Genenncher B, Wirthmueller L, Roth C, Klenke M, Ma L, Sharon A, Wiermer M. Nucleoporin-regulated MAP kinase signaling in immunity to a necrotrophic fungal pathogen. Plant Physiol. 2016;172(2):1293-305.

12. Jiang $S$, Xiao L, Huang $P$, Cheng Z, Chen F, Miao Y, Fu YF, Chen Q, Zhang $X M$. Nucleoporin Nup98 participates in flowering regulation in a CONSTANS-independent mode. Plant Cell Rep. 2019;38(10):1263-71.

13. Gan S, Amasino RM. Making sense of senescence (molecular genetic regulation and manipulation of leaf senescence). Plant Physiol. 1997;113(2): 313-9.

14. Buchanan-Wollaston $\mathrm{V}$. The molecular biology of leaf senescence. J Exp Bot. 1997:48(307):181-99.

15. Kroger R, Holland MM, Moore MT, Cooper CM. Plant senescence: a mechanism for nutrient release in temperate agricultural wetlands. Environ Pollut. 2007;146(1):114-9. 
16. Himelblau E, Amasino RM. Nutrients mobilized from leaves of Arabidopsis thaliana during leaf senescence. J Plant Physiol. 2001;158:1317-23.

17. Streb S, Zeeman SC. Starch metabolism in Arabidopsis, vol. 10. Rockville: The Arabidopsis book / American Society of Plant Biologists; 2012. p. e0160.

18. Ruan YL. Sucrose metabolism: gateway to diverse carbon use and sugar signaling. Annu Rev Plant Biol. 2014;65:33-67.

19. van Doorn WG. Is the onset of senescence in leaf cells of intact plants due to low or high sugar levels? J Exp Bot. 2008;59(8):1963-72.

20. Wingler A, Delatte TL, O'Hara LE, Primavesi LF, Jhurreea D, Paul MJ, Schluepmann $\mathrm{H}$. Trehalose 6-phosphate is required for the onset of leaf senescence associated with high carbon availability. Plant Physiol. 2012; 158(3):1241-51.

21. Sheen J. Master regulators in plant glucose signaling networks. J Plant Biol. 2014;57(2):67-79.

22. Thomas H. Senescence, ageing and death of the whole plant. New Phytol. 2013;197(3):696-711

23. Wingler A, Purdy S, MacLean JA, Pourtau N. The role of sugars in integrating environmental signals during the regulation of leaf senescence. J Exp Bot. 2006:57(2):391-9.

24. Cho Jl, Ryoo N, Eom JS, Lee DW, Kim HB, Jeong SW, Lee YH, Kwon YK, Cho $\mathrm{MH}$, Bhoo SH, et al. Role of the rice hexokinases OsHXK5 and OsHXK6 as glucose sensors. Plant Physiol. 2009;149(2):745-59.

25. Baena-Gonzalez E, Rolland F, Thevelein JM, Sheen J. A central integrator of transcription networks in plant stress and energy signalling. Nature. 2007; 448(7156):938-42.

26. Xiong Y, Sheen J. Rapamycin and glucose-target of rapamycin (TOR) protein signaling in plants. J Biol Chem. 2012;287(4):2836-42.

27. Xiong Y, Sheen J. Moving beyond translation: glucose-TOR signaling in the transcriptional control of cell cycle. Cell Cycle. 2013;12(13):1989-90.

28. D'Angelo MA, Raices M, Panowski SH, Hetzer MW. Age-dependent deterioration of nuclear pore complexes causes a loss of nuclear integrity in postmitotic cells. Cell. 2009;136(2):284-95.

29. Webster BM, Colombi P, Jager J, Lusk CP. Surveillance of nuclear pore complex assembly by ESCRT-IIINps4. Cell. 2014;159(2):388-401.

30. Fichtman B, Harel A. Stress and aging at the nuclear gateway. Mech Ageing Dev. 2014;135:24-32

31. Fernandez-Martinez J, Rout MP. Nuclear pore complex biogenesis. Curr Opin Cell Biol. 2009;21(4):603-12.

32. Hetzer MW. The role of the nuclear pore complex in aging of post-mitotic cells. Aging (Albany NY). 2010;2(2):74-5.

33. Kim SY, Kang HT, Choi HR, Park SC. Reduction of Nup107 attenuates the growth factor signaling in the senescent cells. Biochem Biophys Res Commun. 2010;401(1):131-6.

34. Snow CJ, Paschal BM. Roles of the nucleoporin Tpr in cancer and aging. Adv Exp Med Biol. 2014;773:309-22.

35. David-Watine B. Silencing nuclear pore protein Tpr elicits a senescent-like phenotype in cancer cells. PLoS One. 2011;6(7):e22423.

36. Xiao L, Liu W, Chen F, Zhang X, Chen Q, Fu Y-F. The phenotype analysis of NUP107-160 subcomplex mutants in arabidopsis. J Agric Sci Technol. 2016; 18(5):54-61. https://doi.org/10.13304/j.nykjdb.2016.065.

37. Balazadeh S, Riano-Pachon DM, Mueller-Roeber B. Transcription factors regulating leaf senescence in Arabidopsis thaliana. Plant Biol (Stuttg). 2008; 10(Suppl 1):63-75.

38. Contento AL, Kim SJ, Bassham DC. Transcriptome profiling of the response of Arabidopsis suspension culture cells to Suc starvation. Plant Physiol. 2004; 135(4):2330-47.

39. Gonzali S, Loreti E, Solfanelli C, Novi G, Alpi A, Perata P. Identification of sugar-modulated genes and evidence for in vivo sugar sensing in Arabidopsis. J Plant Res. 2006;119(2):115-23.

40. Miao Y, Smykowski A, Zentgraf U. A novel upstream regulator of WRKY53 transcription during leaf senescence in Arabidopsis thaliana. Plant Biol (Stuttg). 2008;10(Suppl 1):110-20.

41. Hensel LL, Grbic V, Baumgarten DA, Bleecker AB. Developmental and agerelated processes that influence the longevity and senescence of photosynthetic tissues in arabidopsis. Plant Cell. 1993;5(5):553-64. https:// doi.org/10.1105/tpc.5.5.553.

42. Lohman KN, Gan S, John MC, Amasino RM. Molecular analysis of natural leaf senescence in Arabidopsis thaliana. Physiol Plant. 1994;92(2):322-8.

43. Usadel B, Nagel A, Thimm O, Redestig H, Blaesing OE, Palacios-Rojas N, Selbig J, Hannemann J, Piques MC, Steinhauser D, et al. Extension of the visualization tool MapMan to allow statistical analysis of arrays, display of corresponding genes, and comparison with known responses. Plant Physiol. 2005;138(3):1195-204.

44. Bassham DC, Laporte M, Marty F, Moriyasu Y, Ohsumi Y, Olsen LJ, Yoshimoto K. Autophagy in development and stress responses of plants. Autophagy. 2006;2(1):2-11.

45. Bassham DC. Methods for analysis of autophagy in plants. Methods. 2015; 75:181-8.

46. Fujiki Y, Yoshikawa Y, Sato T, Inada N, Ito M, Nishida I, Watanabe A. Darkinducible genes from Arabidopsis thaliana are associated with leaf senescence and repressed by sugars. Physiol Plant. 2001;111(3):345-52.

47. Noh YS, Amasino RM. Regulation of developmental senescence is conserved between Arabidopsis and Brassica napus. Plant Mol Biol. 1999; 41(2):195-206.

48. Weaver LM, Gan S, Quirino B, Amasino RM. A comparison of the expression patterns of several senescence-associated genes in response to stress and hormone treatment. Plant Mol Biol. 1998;37(3):455-69.

49. Klimesova J, Nobis MP, Herben T. Senescence, ageing and death of the whole plant: morphological prerequisites and constraints of plant immortality. New Phytol. 2015;206(1):14-8.

50. Lim PO, Kim HJ, Nam HG. Leaf senescence. Annu Rev Plant Biol. 2007;58: 115-36.

51. Khan $M$, Rozhon W, Poppenberger $B$. The role of hormones in the aging of plants - a mini-review. Gerontology. 2014;60(1):49-55.

52. Guo Y. Towards systems biological understanding of leaf senescence. Plant Mol Biol. 2013;82(6):519-28.

53. Guiboileau A, Sormani R, Meyer C, Masclaux-Daubresse C. Senescence and death of plant organs: nutrient recycling and developmental regulation. C R Biol. 2010;333(4):382-91.

54. Zhang H, Zhou C. Signal transduction in leaf senescence. Plant Mol Biol. 2013;82(6):539-45.

55. Jibran R, Hunter DA, Dijkwel PP. Hormonal regulation of leaf senescence through integration of developmental and stress signals. Plant Mol Biol. 2013;82:547-61.

56. Bakshi M, Oelmuller R. WRKY transcription factors: Jack of many trades in plants. Plant Signal Behav. 2014;9(2):e27700.

57. Koyama T. The roles of ethylene and transcription factors in the regulation of onset of leaf senescence. Front Plant Sci. 2014;5:650.

58. Hinderhofer $\mathrm{K}$, Zentgraf $U$. Identification of a transcription factor specifically expressed at the onset of leaf senescence. Planta. 2001;213(3):469-73.

59. Miao $Y$, Laun T, Zimmermann P, Zentgraf U. Targets of the WRKY53 transcription factor and its role during leaf senescence in Arabidopsis. Plant Mol Biol. 2004;55(6):853-67.

60. Wingler A, Masclaux-Daubresse C, Fischer AM. Sugars, senescence, and ageing in plants and heterotrophic organisms. J Exp Bot. 2009;60(4):1063-6.

61. Lastdrager J, Hanson J, Smeekens S. Sugar signals and the control of plant growth and development. J Exp Bot. 2014;65(3):799-807.

62. Kim JA, Kim HS, Choi SH, Jang JY, Jeong MJ, Lee SI. The importance of the circadian clock in regulating plant metabolism. Int J Mol Sci. 2017;18(12):2680.

63. Seluzicki A, Burko Y, Chory J. Dancing in the dark: darkness as a signal in plants. Plant Cell Environ. 2017;40(11):2487-501.

64. Flis A, Mengin V, Ivakov AA, Mugford ST, Hubberten HM, Encke B, Krohn N, Hohne M, Feil R, Hoefgen R, et al. Multiple circadian clock outputs regulate diel turnover of carbon and nitrogen reserves. Plant Cell Environ. 2019;42(2): 549-73.

65. Kim H, Kim HJ, Vu QT, Jung S, McClung CR, Hong S, Nam HG. Circadian control of ORE1 by PRR9 positively regulates leaf senescence in Arabidopsis. Proc Natl Acad Sci U S A. 2018;115(33):8448-53.

66. Kikis EA, Khanna R, Quail PH. ELF4 is a phytochrome-regulated component of a negativefeedback loop involving the central oscillator components CCA1 and LHY. Plant J. 2005:44(2):300-13.

67. Murashige T, Skoog F. A revised medium for rapid growth and bioassays with tobacco tissue cultures. Physiol Plant. 1962;15:473-97.

68. Wang X, Fan C, Zhang X, Zhu J, Fu YF. BioVector, a flexible system for gene specific-expression in plants. BMC Plant Biol. 2013;13(1):198.

69. Clough SJ, Bent AF. Floral dip: a simplified method for agrobacteriummediated transformation of Arabidopsis thaliana. Plant J. 1998;16(6):735-43.

70. Xiao C, Chen F, Yu X, Lin C, Fu YF. Over-expression of an AT-hook gene, $\mathrm{AHL} 22$, delays flowering and inhibits the elongation of the hypocotyl in Arabidopsis thaliana. Plant Mol Biol. 2009;71(1-2):39-50.

71. Gutierrez L, Mauriat M, Guenin S, Pelloux J, Lefebvre JF, Louvet R, Rusterucci C, Moritz T, Guerineau F, Bellini C, et al. The lack of a systematic validation 
of reference genes: a serious pitfall undervalued in reverse transcriptionpolymerase chain reaction (RT-PCR) analysis in plants. Plant Biotechnol J. 2008;6(6):609-18.

72. Czechowski T, Stitt M, Altmann T, Udvardi MK, Scheible WR. Genome-wide identification and testing of superior reference genes for transcript normalization in Arabidopsis. Plant Physiol. 2005;139(1):5-17.

73. Caspar T, Lin TP, Kakefuda G, Benbow L, Preiss J, Somerville C. Mutants of Arabidopsis with altered regulation of starch degradation. Plant Physiol. 1991;95(4):1181-8.

\section{Publisher's Note}

Springer Nature remains neutral with regard to jurisdictional claims in published maps and institutional affiliations.

Ready to submit your research? Choose BMC and benefit from:

- fast, convenient online submission

- thorough peer review by experienced researchers in your field

- rapid publication on acceptance

- support for research data, including large and complex data types

- gold Open Access which fosters wider collaboration and increased citations

- maximum visibility for your research: over $100 \mathrm{M}$ website views per year

At BMC, research is always in progress.

Learn more biomedcentral.com/submissions 